\title{
Nonlinear Time-Varying Turbulence Signal Processing and Simulation Under Curvilinear Coordinate System
}

\author{
Xiaoyang Liu ${ }^{1,2}$, Wanping Liu ${ }^{1}$, Chao Liu ${ }^{1}$, Xiaoping Zeng ${ }^{2}$ \\ ${ }^{1}$ School of Computer Science and Engineering, Chongqing University of Technology, Chongqing, China \\ ${ }^{2}$ Postdoctoral Research Station of Information and Communication, Engineering, Chongqing University, Chongqing, China
}

Email address:

1xy3103@163.com (Xiaoyang Liu)

\section{To cite this article:}

Xiaoyang Liu, Wanping Liu, Chao Liu, Xiaoping Zeng. Nonlinear Time-Varying Turbulence Signal Processing and Simulation Under Curvilinear Coordinate System. Communications. Vol. 4, No. 1, 2016, pp. 1-7. doi: 10.11648/j.com.20160401.11

Received: April 16, 2016; Accepted: April 25, 2016; Published: May 13, 2016

\begin{abstract}
In order to analyse the nature of the turbulence, the curvilinear coordinate system is established according to the relations of cartesian coordinate system. Aiming at this turbulence mechanism, the turbulence boundary equations were established, then all grid points of the coordinates can be got by the established equations. The dangerous degree measure (risk factor) was put forward. The mathematical model of risk factor was established as to quantitatively describe the danger level of turbulence. A turbulence signal processing algorithm is proposed combined with the Doppler effect and the computing grid. The simulation results show that the curvilinear coordinate system and three-dimensional turbulence field can reflect the characteristics of turbulence, and risk factor can reflect the danger level of turbulence, the proposed turbulence signal processing algorithm can detect and forecast the turbulence effectively.
\end{abstract}

Keywords: Turbulence, Curvilinear Coordinate System, Grid Generation, Spectrum Width, Wind Velocity

\section{Introduction}

Turbulence has been recognized as a cause of aircraft crashes for some time.

Turbulence refers to the wind speed fluctuations in a short period time. Evoked a lot of In order to understand the nature of the proposed turbulence model, a description of microbursts is necessary. The necessary description and the characteristics of the JAWS (Jamming and Warning System) data sets are included in this paper.

These descriptions contain a discussion of some turbulence models. In anticipation of the need for adding turbulence to the JAWS data for the purpose of flight simulation, relevant aspects of turbulence theory are presented $[1,2,3,4]$. The rule of turbulence motion is irregularity and instability. As for the weather radar, turbulence is the meteorological goal of large particles which speed suddenly change greatly.

The wind loading of a tracking radar antenna, for example, results from a meanvelocity component that varies with time plus superimposed random gusts. In the turbulence region, because of the great variation of river air velocity along the direction of change is quite sharp. Cases like this not only create dangers for the airplane but in danger of life [5, 6, 7].

The classic turbulence models of Von Karman and Dryden has been attracting the people's attention. But these models do not consider the influence of asymmetric wind field. Kolmogorov and other people have realized the no characteristic scales of turbulence $[8,9,10]$. They think that the number of Reynold is large, turbulence is made up of all kinds of different scales of vortex. Maelstrom is produced by boundary condition and instability. Maelstrom is broken into smaller spiral by inertia and energy delivery. The difficulty of turbulence research is that it contains large and small scales of turbulence vortex.

Due to the scale self-similarity of the wavelet transform. Bezerra and Ashrafian used the method of wavelet transform to study the turbulence, its main idea is to use of the domain is transform the space domain into $k$ domain by using Fourier transform. Looking for $k$ value when the signal is the strongest, and to determine the main ingredients or cycle of the signal. So, they can decide the signal is strong or not, or in which scale the signal is the strongest $[11,12]$. This approach does not play the advantages of wavelet transform. Because the wavelet transform is related to the time factor, but Fourier transform doesn't have the time factor. 
Turbulence is a highly complicated nonlinear dissipative system. The MLSD (Maximum-likelihood Sequence Detection) is realized in lognormal statistical model of the atmospheric turbulence by Susumu. G and Philippe R [13, 14]. But MLSD cannot track the time change of the multiplicative noise statistics. The influences of turbulence detection is researched with PPP (pulse pair processing) algorithm by Y. $\mathrm{Li}$ and V. Raj Mohan [15, 16]. References [17, 18, 19, 20] researched the autocorrelation characteristics of the Von Karman model, but they have not considered the effect of turbulence scale factors. References [21, 22, 23] mainly studied the numerical simulation of atmospheric turbulence, Gaussian noise sequence is dealt by adopting a double random exchange of minimization method. The method will vastly improve the degree of bleaching, but there is no discussion the wind speed estimation of turbulence flow field. Those studies have been important for turbulence detecting, and in particular their the frequency spectrum analysis of turbulence [24, 25].

Depending upon the root mean square of turbulence Doppler velocity $\sigma_{V}$, turbulence level classification can be defined in Table 1.

Table 1. Turbulence level classification.

\begin{tabular}{lllll}
\hline turbulence intensity & negligible & slight & moderate & strong \\
\hline$\sigma_{V}(\mathrm{~m} / \mathrm{s})$ & $0-1.5$ & $1.5-3$ & $3-4.5$ & $>4.5$ \\
\hline
\end{tabular}

The sketch of turbulence observation under airborne weather radar is shown in Figure 1.



Figure 1. The sketch of turbulence observation.

In Figure 1 . The plane fly at a constant velocity $V$ along $\mathrm{X}$ axis. The antenna angle of view is $\alpha_{a}$. from the scatterer $\mathrm{S}$ return of the received signal The Doppler frequency shift of the received signal from the scatterer $\mathrm{S}$ is described by

$$
f(\alpha)=\frac{2 V}{\lambda} \cos \alpha
$$

Where $\lambda$ is the wavelength of airborne weather radar. $\alpha$ is the azimuth of Scatterer S.

\section{Curvilinear Coordinate System}

For convenience, cartesian coordinate system $x-y-z$ is written as $y^{1}, y^{2}, y^{3}$, and cylindrical coordinate system $r-\varphi-z$ is written as $x^{1}, x^{2}, x^{3}$. The mutual conversion relationship of the two coordinate systems can be expressed as

$$
\begin{aligned}
& \left\{\begin{array}{l}
x^{1}=\sqrt{\left(y^{1}\right)^{2}+\left(y^{2}\right)^{2}}=x^{1}\left(y^{1}, y^{2}\right) \\
x^{2}=\arctan \frac{y^{2}}{y^{1}} x^{2}\left(y^{1}, y^{2}\right) \\
x^{3}=y^{3}=x^{3}\left(y^{3}\right)
\end{array}\right. \\
& \left\{\begin{array}{l}
y^{1}=x^{1} \cos x^{2}=y^{1}\left(x^{1}, x^{2}\right) \\
y^{2}=x^{1} \sin x^{2}=y^{2}\left(x^{1}, x^{2}\right) \\
y^{3}=x^{3}=y^{3}\left(x^{3}\right)
\end{array}\right.
\end{aligned}
$$

It can be seen that the relations between the two set of functions is single-valued continuous function. The two Jacobian determinant of coordinate transformation are not zero, in addition to the $(0,0,0)$ coordinate. In a certain space area, Jacobian determinant can be described by

$$
J^{\prime}=\frac{\partial\left(x^{1}, x^{2}, x^{3}\right)}{\partial\left(y^{1}, y^{2}, y^{3}\right)} \neq 0 ; J^{\prime}=\frac{\partial\left(y^{1}, y^{2}, y^{3}\right)}{\partial\left(x^{1}, x^{2}, x^{3}\right)} \neq 0
$$

In the region $\mathrm{D}$, there is one-to-one correspondence between a $\operatorname{dot} P$ and an array $\left(x^{1}, x^{2}, x^{3}\right)$. Take $x^{1}, x^{2}, x^{3}$ as the coordinate of the $\operatorname{dot} P$. So, a curvilinear coordinate system is established [26, 27, 28], which is shown in Figure 2.



Figure 2. Curvilinear coordinate system in region D.

\section{The Generation of Turbulence Calculation Grid}

Now make $\mathrm{d} m$ represents the quality of the fluid micelle, $\rho$ shows the average density of the fluid micelle, $\mathrm{d} \tau$ expresses the volume of the fluid micelle. $V$ is absolute velocity.

Based on the mass conservation law, the energy conservation law and the definition of divergence. When the velocity field is continuously and differentiable, we have

$$
\frac{\partial \rho}{\partial t}+\nabla \cdot(\rho V)=0
$$

The motion equations of fluid micelle is described by 


$$
\begin{gathered}
\rho \frac{\mathrm{D} \boldsymbol{V}}{\mathrm{D} t}=\rho \boldsymbol{F}+\nabla . \boldsymbol{\Pi}=\rho \boldsymbol{F}-\nabla \rho+\boldsymbol{f}_{\tau}\left(\boldsymbol{f}_{\tau}=\nabla . \boldsymbol{\Pi}^{\prime}\right) \\
\boldsymbol{\Pi}=2 \mu \varepsilon-\left[p+\frac{2}{3} \mu(\nabla \cdot \boldsymbol{V})\right] \cdot \boldsymbol{I} \\
\boldsymbol{\Pi}^{\prime}=2 \mu \varepsilon-\frac{2}{3} \mu(\nabla \cdot \boldsymbol{V}) \cdot \boldsymbol{I}
\end{gathered}
$$

In the above formulas, $\boldsymbol{F}$ is the mass force of the unit mass fluid and inertial force. $\boldsymbol{\Pi}$ expresses the stress tensor. $\boldsymbol{f}_{\tau}$ denotes the viscous force. $\mu$ is a proportionality constant associated with the viscosity of fluid. $\boldsymbol{I}$ is a unit vector.

Under the $\xi, \eta, \zeta$ curve coordinate system, a two-dimensional curve coordinate system can be derived according to the fluid mechanics in combination with Lame coefficient.

$$
\left\{\begin{array}{l}
\xi=\xi(x, y) \\
\eta=\eta(x, y)
\end{array}\right.
$$

A set of equations can be worked out of meeting with some formulas and other boundary conditions.

$$
\left\{\begin{array}{l}
x=x(\xi, \eta) \\
y=y(\xi, \eta)
\end{array}\right.
$$

The coordinates of all grid points $(x, y)$ can be attained associated with $\xi, \eta=1,2,3, \cdots$. Then connect these grid points by the smooth curve, the grid lines of curve coordinate can be got by connecting these grid points with the smooth curve.

\section{Turbulence Signal Processing and Detecting}

\subsection{The Risk Factor Model}

To conduct quantitative analysis on the risk of turbulence, a risk factor $F$ for danger judgment is proposed. According to the theorem of quality continuity, a risk factor $F$ can be defined when the vertical wind speed is proportional to the horizontal divergence in the given the height.

$$
F \approx \frac{v_{g}}{\mathrm{~g}} \frac{\partial v_{x}}{\partial \mathrm{x}}-h \cdot \frac{\frac{\partial v_{x}}{\partial \mathrm{x}}+\frac{v_{x}}{R}}{v_{g}}
$$

In the above formula, $v_{g}$ is the ground speed of aircraft. $\mathrm{g}=9.8 \mathrm{~m} / \mathrm{s}^{2} . W_{x}$ expresses the horizontal wind velocity. $\frac{\partial v_{x}}{\partial \mathrm{x}}$ is the rate of change of horizontal wind velocity. $h$ denotes the height of aircraft. $R$ is the distance from the airplane to the center of the micro air flow. The positive value of risk factor expresses the descended performance of airplane. The negative value of risk factor denotes the increased performance of airplane.

\subsection{Three-Dimensional Turbulence Field}

In order to meet the demands of numerical simulation. The turbulence field in the space is divided into $M_{1} \times M_{2} \times M_{3}$ grid points. The Three-dimensional turbulence field is shown in Figure 3.

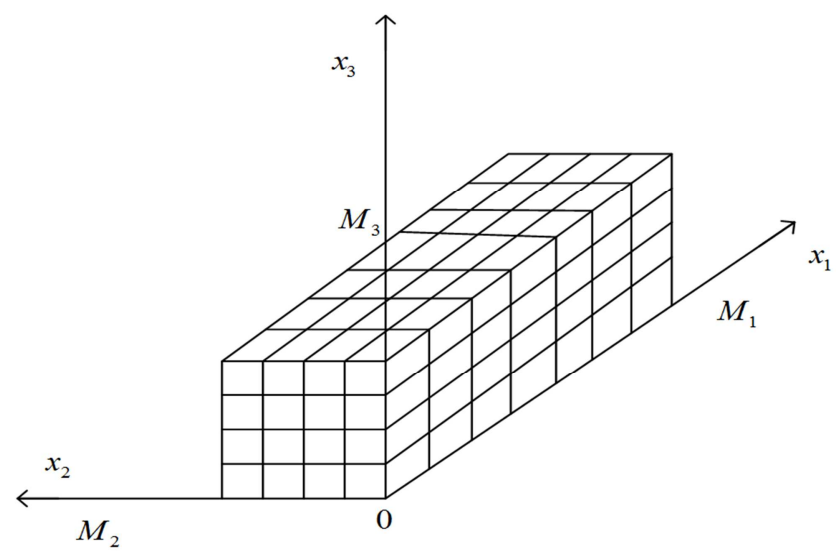

Figure 3. Three-dimensional turbulence field.

According to the nature of FFT transform, the two relations between the space sampling rate of time domain and the sampling rate of frequency domain can be described by

$$
\begin{array}{cc}
\Delta v_{i}=\frac{v_{s_{i}}}{M_{i}}, & i=1,2,3 \\
\Delta r_{i}=\frac{1}{v_{s_{i}}}, & i=1,2,3
\end{array}
$$

In the above formulas, $v_{s_{i}}$ is the sampling rate of time domain in the direction $r_{i} . M_{i}$ is the number of grid points in the direction $r_{i} . \Delta v_{i}$ is the grid spacings of frequency domain in the direction $r_{i} . \Delta r_{i}$ is the grid spacings of time domain in the direction $r_{i}$.

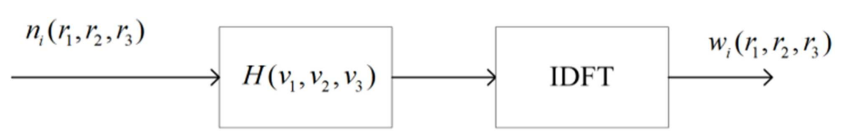

Figure 4. The generation of turbulence field.

In Figure 4, $n_{i}\left(r_{1}, r_{2}, r_{3}\right)$ is the three road of Gaussian white noise sequence, $i=1,2,3 . H\left(v_{1}, v_{2}, v_{3}\right)$ denotes the three dimensional formed filter function. IDFT expresses the inverse transform of three dimensional discrete Fourier. $w_{i}\left(r_{1}, r_{2}, r_{3}\right)$ shows the isotropic turbulence.

\subsection{Turbulence Signal Processing}

The three dimensional discrete Fourier transform and its inverse transform can be expressed as 


$$
\begin{gathered}
X_{k_{1}, k_{2}, k_{3}}=\sum_{n_{1}=0}^{M_{1}-1} \sum_{n_{2}=0}^{M_{2}-1} \sum_{n_{3}=0}^{M_{3}-1} x_{n_{1}, n_{2}, n_{3}} \\
\cdot \mathrm{e}^{-\mathrm{j} 2 \pi\left(n_{1} k_{1} / M_{1}+n_{2} k_{2} / M_{2}+n_{3} k_{3} / M_{3}\right)} \\
x_{n_{1}, n_{2}, n_{3}}=\frac{1}{M_{1} M_{2} M_{3}} \sum_{k_{1}=0}^{M_{1}-1} \sum_{k_{2}=0}^{M_{2}-1} \sum_{k_{3}=0}^{M_{3}-1} X_{k_{1}, k_{2}, k_{3}} \\
\cdot \mathrm{e}^{+\mathrm{j} 2 \pi\left(n_{1} k_{1} / M_{1}+n_{2} k_{2} / M_{2}+n_{3} k_{3} / M_{3}\right)}
\end{gathered}
$$

In the above formulas, $n_{i}=0,1, \cdots, M_{i}-1$; $k_{i}=0,1, \cdots, M_{i}-1 . \quad x_{n_{1}, n_{2}, n_{3}}$ is the numerical sequence of time domain of three dimensional Fourier transformation. $X_{k_{1}, k_{2}, k_{3}}$ is the numerical sequence of frequency domain of three dimensional Fourier transformation.

The time domain noise variance of the three dimensional zero mean is $\sigma_{x_{n}}^{2}$. So the frequency domain noise variance of the three dimensional zero mean can be described by

$$
\begin{gathered}
\sigma_{N_{k}}^{2}=\mathrm{E}\left[X_{k_{1}, k_{2}, k_{3}} \cdot X_{k_{1}, k_{2}, k_{3}}^{*}\right]=M_{1} M_{2} M_{3} \sigma_{x_{n}}^{2}\left(\Delta r_{1} \Delta r_{2} \Delta r_{3}\right)^{2} \\
=M_{1} M_{2} M_{3} \sigma_{x_{n}}^{2}\left(v_{s 1} v_{s 2} v_{s 3}\right)^{2}
\end{gathered}
$$

The following formulas can be got by the FFT of three-dimensional symmetrical characteristic.

$$
\begin{gathered}
X\left(M_{1}-k_{1}, 0,0\right)=X^{*}\left(k_{1}, 0,0\right) \\
\operatorname{Im}\left[X\left(M_{1} / 2,0,0\right)\right]=0 \\
X\left(M_{1}-k_{1}, M_{2}-k_{2}, M_{3}-k_{3}\right)=X^{*}\left(k_{1}, k_{2}, k_{3}\right) \\
\operatorname{Im}\left[X\left(M_{1} / 2, M_{2} / 2, M_{3} / 2\right)\right]=0
\end{gathered}
$$

In turbulence wind field, the echo signal sampling sequence $C(n)$ of a certain range bin can be described by

$$
C(n)=A(n)+j B(n)
$$

$A(n)$ and $B(n)$ represent the in-phase and quadrature components of pulse sampling signal, respectively.

The power spectrum of echo signal can be got

$$
\begin{aligned}
& P\left(f_{i}\right)=\left|\sum_{n=0}^{N-1} M(n) \exp \left(-j 2 \pi \frac{n i}{N}\right)\right|^{2} \\
& f_{i}=\frac{i}{N T_{s}}, i=0,1 \ldots \ldots N-1
\end{aligned}
$$

In the above formula. $N$ is the length of sampling sequence. $T_{s}$ denotes the sampling interval of echo signal. $M(n)$ expresses the amplitude.
The average Doppler speed $V_{\text {ave }}$ and spectrum width $W_{\text {spe }}$ of echo signal can be described by

$$
\begin{aligned}
V_{\text {ave }}= & \frac{\sum_{i=0}^{N-1} f_{i} P\left(f_{i}\right)}{\sum_{i=0}^{N-1} P\left(f_{i}\right)} \\
W_{\text {spe }}= & \frac{\sum_{i=0}^{N-1}\left(f_{i}-V_{\text {ave }}\right)^{2} P\left(f_{i}\right)}{\sum_{i=0}^{N-1} P\left(f_{i}\right)}
\end{aligned}
$$

The echo signal of turbulence processing is shown in Figure 5.

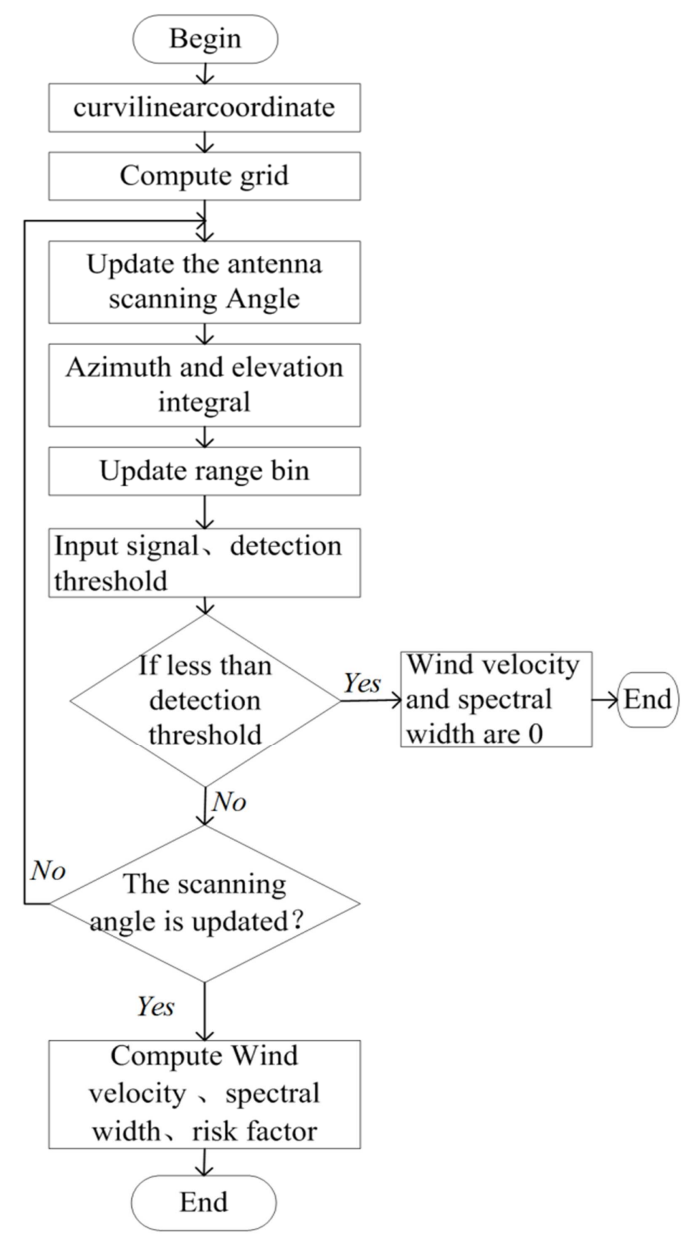

Figure 5. The echo signal of turbulence processing flow chart.

\section{Simulation Results Analysis}

The echo signal of three-dimensional power spectrum Doppler velocity of turbulence echo signal is shown in Figure 6. It can be seen that the proposed algorithm can estimate the wind speed distribution of the turbulence wind field effectively. 


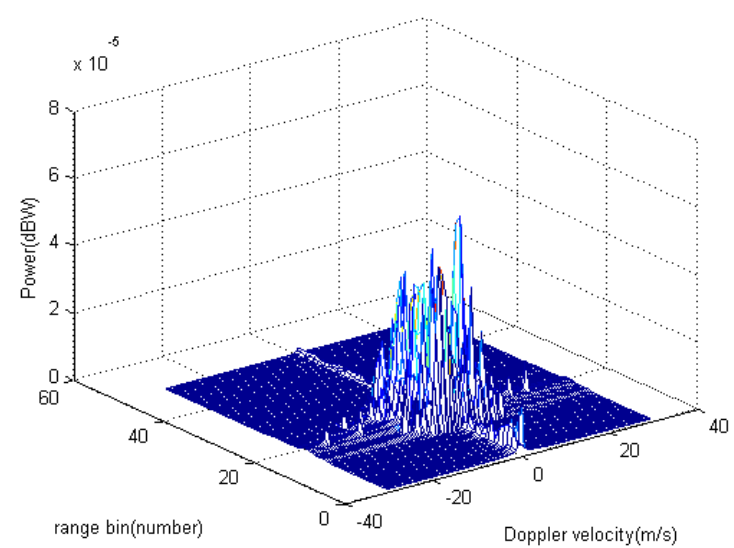

Figure 6. Three-dimensional power spectrum analysis.

In order to verify the validity of the algorithm, we compare the real wind velocity and the proposed algorithm. As can be seen from the Figure 7, the wind speed can be accurately estimated by the proposed algorithm.

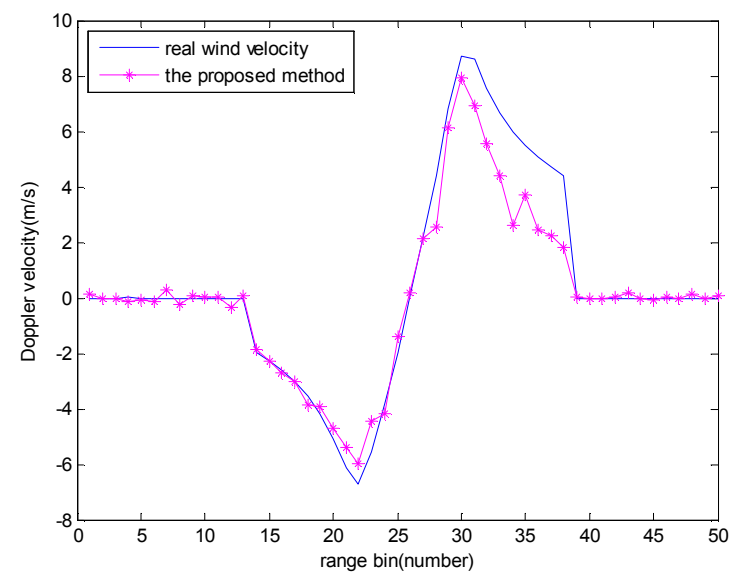

Figure 7. The Doppler velocity in different range bin.

When the turbulence signal is processed by airborne weather radar, firstly estimate the spectrum width of echo signal in wind filed with different range bins, in order to determine whether the target echo is turbulence or not. Figure 8 and Figure 9 are the risk factor analysis of turbulence in different range bins.



Figure 8. The risk factor analysis of turbulence (have ground clutter).

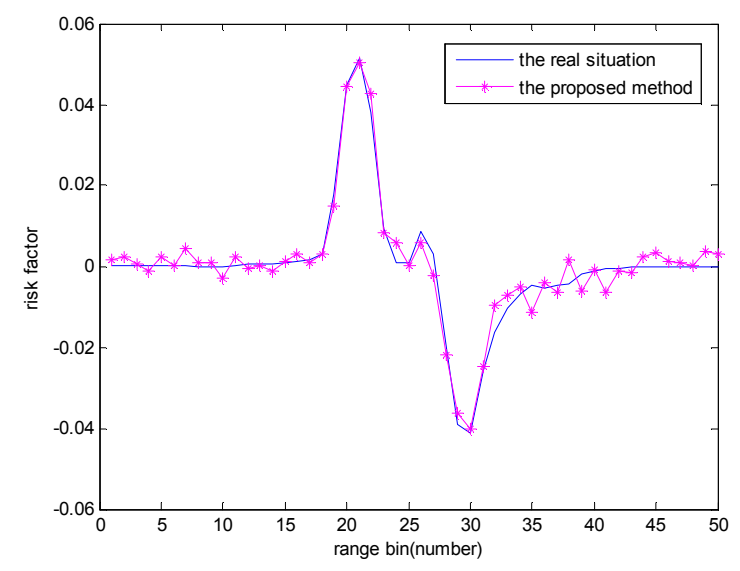

Figure 9. The risk factor analysis of turbulence (no ground clutter).

As can be seen from Figure 8 and Figure 9, the risk factor is different in different range bins, the risk factor reaches the maximum between the 20th rang bin and the 25 th range bin. The performance of no ground clutter is better than the situation of have ground clutter through the Figure 8 and Figure 9.

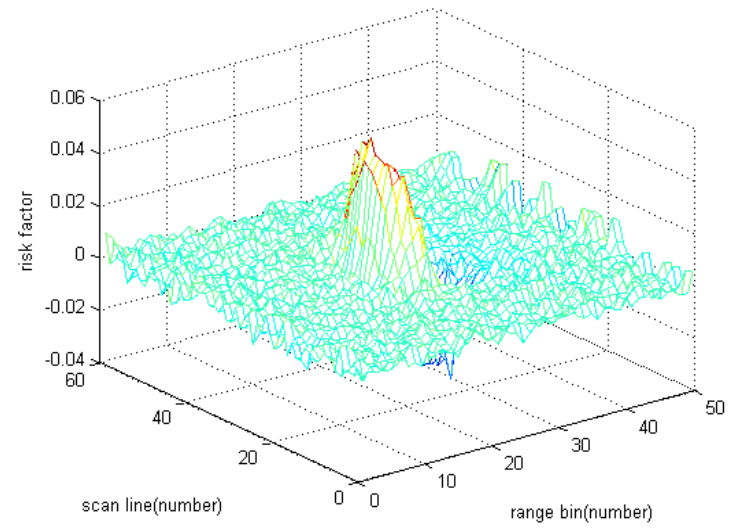

Figure 10. Three dimensional risk factor analysis.

The diagram in Figure 10 shows the three dimensional risk factor in different scanning lines. The Figure11 expresses the risk factor in different range bin. According to risk factor definition, the threshold value of risk factor can be set to 0.2 . It is dangerous in some areas as can be seen from Figure 11.

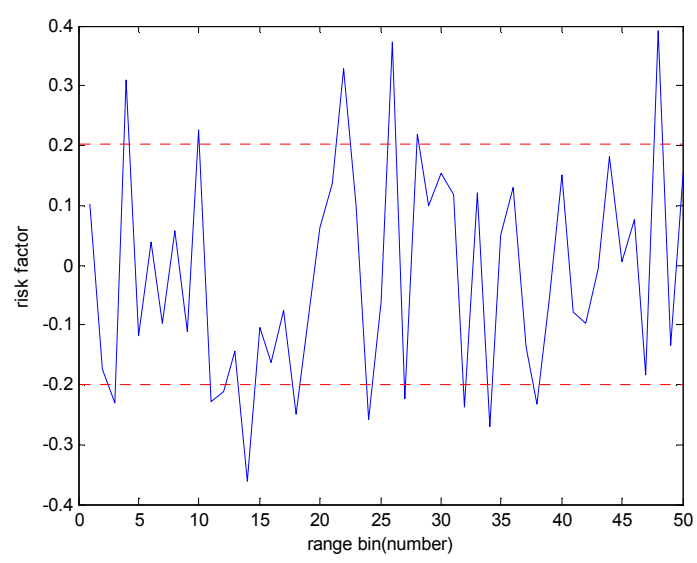

Figure 11. The risk factor in different range bin. 


\section{Conclusions}

In this paper, a curvilinear coordinate system is established in this paper. The mathematical model of turbulence calculation grid is set up combined with the continuous equation and motion equation of turbulence. The grid computing model is good for subdivision turbulence field. The grid could enable detailed simulations of turbulence. In order to be able to quantitative analysis the harm of turbulence, the risk factor of turbulence is set up which reflects hazard of turbulence. A turbulence signal algorithm is proposed combined with Doppler effect. This algorithm can reflect the characteristics of turbulence and can carry on the detection and prediction of turbulence.

This turbulence signal processing model and mathematical model of risk factor can be used as the reference model of microburst wind shear. Considering the nonlinear of turbulence, the space-time adaptive processing and deconvolution method can be used for turbulence research and other characteristics.

\section{Acknowledgment}

The paper are supported by Chongqing Postdoctoral Researchers Research Project Special Funded (Grant No. Xm2015029), Scientific and Technological Research Program of Chongqing Municipal Education Commission (Grant No. KJ1500926, KJ1500904, KJ1500920), Chongqing Science and Technology Committee Fund of Foundation and Cutting-edge Research Plan (Grant No. cstc2014jcyjA40007), Natural Science Foundation of China (Grant No. 61502064, 61503052, 91438104, 11547148).

\section{References}

[1] Oreifej. O, Xin-Li, Shah. M, Simultaneous Video Stabilization and Moving Object Detection in Turbulence, IEEE Transactions on Pattern Analysis and Machine Intelligence, 2013, 35 (2): 450-462.

[2] Treib. M, Burger. K, Reichl. F, Meneveau. C, Szalay. A, Westermann. R, Turbulence Visualization at the Terascale on Desktop PCs, IEEE Transactions on Visualization and Computer Graphics, 2012, 18 (2): 2169-2177.

[3] Gil. E, Laguna. P, Martinez. J. P, Barquero-Perez. O, Garcia-Alberola. A, Sornmo. L, Heart Rate Turbulence Analysis Based on Photoplethysmography, IEEE Transactions on Biomedical Engineering, 2013, 60 (11): 3149-3155.

[4] Gibson. K. B, Nguyen. T. Q, An Analysis and Method for Contrast Enhancement Turbulence Mitigation, IEEE Transactions on Image Processing, 2014, 23 (7): 3179-3190.

[5] Xuan Tang, Zhaocheng Wang, Zhengyuan Xu, Ghassemlooy. Z, Multihop Free-Space Optical Communications Over Turbulence Channels with Pointing Errors using Heterodyne Detection, Journal of Lightwave Technology, 2014, 32 (15): 2597-2604.

[6] Rajbhandari. S, Ghassemlooy. Z, Haigh. P. A, Kanesan. T, Xuan Tang, Experimental Error Performance of Modulation Schemes
Under a Controlled Laboratory Turbulence FSO Channel, Journal of Lightwave Technology, 2015, 33 (1): 244-250.

[7] Pham. H. T. T, Dang. N. T, Pham. A. T, Effects of atmospheric turbulence and misalignment fading on performance of serial-relaying M-ary pulse-position modulation free-space optical systems with partially coherent Gaussian beam, IET Communications, 2014, 8 (10): 1762-1768.

[8] Puryear. A. L, Shapiro. J. H, Parenti. R. R, Reciprocity-enhanced optical communication through atmospheric turbulence - Part II: Communication architectures and performance, IEEE/OSA Journal of Optical Communications and Networking, 2013, 5 (8): 888-900.

[9] Kaur. P, Jain. V. K, Kar. S, Performance Analysis of FSO Array Receivers in Presence of Atmospheric Turbulence, IEEE Photonics Technology Letters, 2014, 26 (2): 1165-1168.

[10] Xuegui Song, Fan Yang, Julian Cheng, Alouini. M. S, BER of Subcarrier MPSK and MDPSK Systems in Atmospheric Turbulence, Journal of Lightwave, 2015, 33 (1): 161-170.

[11] Vu. B. T, Dang. N. T, Thang. T. C, Pham. A. T, Bit error rate analysis of rectangular QAM/FSO systems using an APD receiver over atmospheric turbulence channels, IEEE/OSA Journal of Optical Communications and Networking, 2013, 5 (5): 437-446.

[12] Katherine Mc Caffrey, Baylor Fox-Kemper, Peter E, Hamlington. Jim Thomson, Characterization of turbulence anisotropy, coherence, and intermittency at a prospective tidal energy site: Observational data analysis, Renewable Energy, 2015, 76 (4): 441-453.

[13] Susumu. G, J. C. Vassilicos, Energy dissipation and flux laws for unsteady turbulence, Physics Letters A, 2015, 379 (16): 1144-1148.

[14] Philippe R, Spalart. Philosophies and fallacies in turbulence modeling, Progress in Aerospace Sciences, 2015, 74: 1-15.

[15] Y. Li, A. M. Castro, T. Sinokrot, W. Prescott, P. M. Carrica, Coupled multi-body dynamics and CFD for wind turbine simulation including explicit wind turbulence, Renewable Energy, 2015, 76, (4): 338-361.

[16] V. Raj Mohan, D. C. Haworth, Turbulence-chemistry interactions in a heavy-duty compression-ignition engine, Proceedings of the Combustion Institute, 2015, 35 (3): 3053-3060.

[17] J. Shinjo, J. Xia, A. Umemura, Droplet/ligament modulation of local small-scale turbulence and scalar mixing in a dense fuel spray, Proceedings of the Combustion Institute, 2015, 35 (2): 1595-1602.

[18] K. Prabu, D. Sriram. Kumar, MIMO free-space optical communication employing coherent BPOLSK modulation in atmospheric optical turbulence channel with pointing errors, Optics Communications, 2015, 343 (15): 188-194.

[19] Erhan Pulat, Hifzı Arda Ersan, Numerical simulation of turbulent airflow in a ventilated room: Inlet turbulence parameters and solution multiplicity, Energy and Buildings, 2015, 93 (15): 227-235.

[20] Andreas Engelen, Susanne Schmidt, Michael Buchsteiner, The Simultaneous Influence of National Culture and Market Turbulence on Entrepreneurial Orientation: A Nine-country Study, Journal of International Management, 2015, 21 (7): 18-30. 
[21] Xiaoyang Liu, Yong Li, Chengyu Feng, Simulation and analysis of turbulence signals in airborne pulse Doppler radar, Systems engineering and electronics, 2015, 21 (1): 18-30.

[22] Xiaoyang Liu, Yong Li, Turbulence signal processing in the airborne weather radar, International Journal of Advancements in Computing Technology, 2013, 34 (5): 816-824.

[23] Yong Li, Xiaoyang Liu, Chengyu Feng, Three dimensional turbulent flow formation and simulation analysis in airborne radar, Systems engineering and electronics, 2013, 35 (6): 1193-1198.

[24] Paolo Orlandi, Sergio Pirozzoli, Matteo Bernardin, George F. Carnevale, A minimal flow unit for the study of turbulence with passive scalars, Journal of Turbulence, 2014, 15 (2): 731-751.
[25] L. Djenidi, S. F. Tardu, R. A. Antonia, L. Danaila, Breakdown of Kolmogorov's first similarity hypothesis in grid turbulence, Journal of Turbulence, 2014, 15 (3): 596-610.

[26] Xiaoyang Liu, Chao Liu. Wanping Liu. Wind Shear Target Echo Modeling and Simulation. Discrete Dynamics in Nature and Society, 2015 (4): 1-6.

[27] Ugur Cakir, Ertugrul Kargi, Hakan Sarman, Cengiz Isik. Impact of Diabetic Foot on Selected Psychological or Social Characteristics. Journal of Diabetes Research, 2014, 8: 2-9.

[28] Detlef Lohse, Siegfried Grossmann. Intermittency in turbulence. Physica A: Statistical Mechanics and its Applications, 1993, 194 (2): 519-531. 